\title{
Recent developments in the line-by-line modeling of outgoing longwave radiation
}

\author{
S.A. Buehler ${ }^{\mathrm{a}, *}$, A. von Engeln ${ }^{\mathrm{a}}$, E. Brocard ${ }^{\mathrm{a}}$, V.O. John ${ }^{\mathrm{a}}$, T. Kuhn ${ }^{\mathrm{b}}$, P. Eriksson ${ }^{\mathrm{c}}$ \\ ${ }^{a}$ Institute of Environmental Physics, University of Bremen, Otto-Hahn-Allee 1, D-28359 Bremen, Germany \\ ${ }^{\mathrm{b}}$ I. Physikalisches Institut, Universitaet zu Koeln, Cologne, Germany \\ ${ }^{\mathrm{c}}$ Department of Radio and Space Science, Chalmers University of Technology, Gothenburg, Sweden
}

\begin{abstract}
High frequency resolution radiative transfer model calculations with the Atmospheric Radiative Transfer Simulator (ARTS) were used to simulate the clear-sky outgoing longwave radiative flux (OLR) at the top of the atmosphere. Compared to earlier calculations by Clough and coworkers the model used a spherical atmosphere instead of a plane parallel atmosphere, updated spectroscopic parameters from HITRAN, and updated continuum parameterizations from Mlawer and coworkers. These modifications lead to a reduction in simulated OLR by approximately $4.1 \%$, the largest part, approximately $2.5 \%$, being due to the absence of the plane parallel approximation. As a simple application of the new model, the sensitivity of OLR to changes in humidity, carbon dioxide concentration, and temperature were investigated for different cloud-free atmospheric scenarios. It was found that for the tropical scenario a $20 \%$ change in humidity has a larger impact than a doubling of the carbon dioxide concentration. The sensitive altitude region for temperature and humidity changes is the entire free troposphere, including the upper troposphere where humidity data quality is poor.

(C) 2005 Elsevier Ltd. All rights reserved.
\end{abstract}

PACS: 42.68.Wt; 42.68.Mj; 41.20.Jb; 84.40.-x

Keywords: Outgoing longwave radiation; OLR; Continua; Infrared; Heating rates; Jacobians; Humidity

\section{Introduction}

The earth and its atmosphere absorb the shortwave (SW) radiation coming from the sun and emit thermal longwave (LW) radiation to space. These two radiation streams can be represented approximately by blackbody radiation of $6000 \mathrm{~K}$ for the solar SW and $290 \mathrm{~K}$ for the terrestrial LW. The balance between the incoming SW radiation and the outgoing LW radiation (OLR) determines the temperature in the atmosphere and on the earth's surface [1-3].

The OLR originates partly from the surface but to a significant part from higher levels of the atmosphere. Because of the lower temperature at these levels, the OLR is reduced compared to a hypothetical earth without

\footnotetext{
*Corresponding author. Tel.: + 494212184417 ; fax: + 494212184555.

E-mail address: sbuehler@uni-bremen.de (S.A. Buehler).

$U R L:$ http://www.sat.uni-bremen.de/.
} 


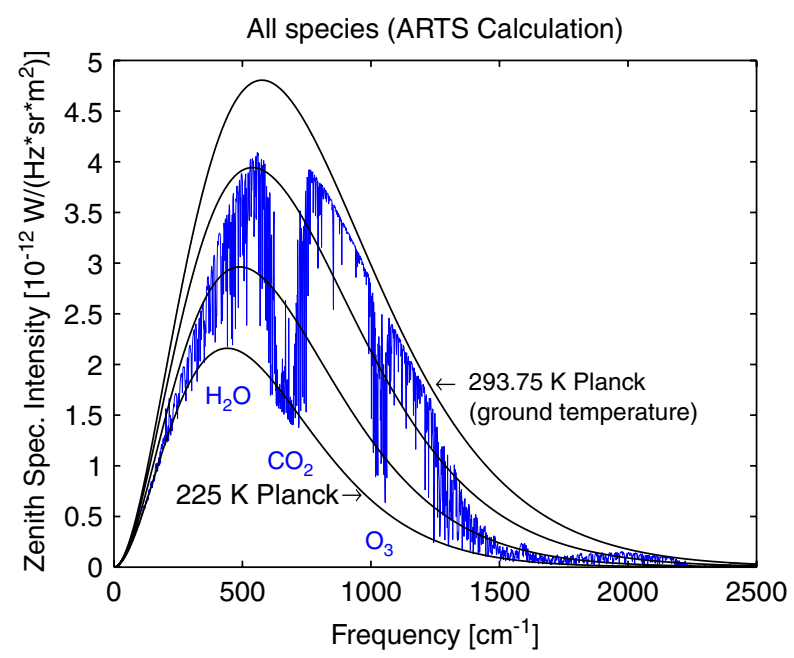

Fig. 1. A radiative transfer model simulation of the TOA zenith monochromatic radiance for a mid-latitude summer atmosphere. Smooth solid lines indicate Planck curves for different temperatures: 225, 250, 275, and 293.75 K. The latter was the assumed surface temperature. The calculated quantity has to be integrated over frequency and direction to obtain total OLR.

atmosphere. Fig. 1 shows a high resolution radiative transfer model simulation of clear-sky monochromatic radiance at the top of the atmosphere (TOA), which illustrates this. Besides the calculated spectrum, it shows Planck curves for different temperatures. An integration over all frequencies and directions yields the OLR. The reduction of OLR compared to a hypothetical earth without atmosphere is of course nothing else than the atmospheric 'greenhouse' effect. From the known incoming solar SW radiation we can easily infer the global average OLR to be close to $240 \mathrm{~W} \mathrm{~m}^{-2}$ because the incoming and outgoing radiation fluxes must balance [2]. However, there is considerable variability for different latitudes and weather conditions, so that local OLR values vary between about 160 and $320 \mathrm{~W} \mathrm{~m}^{-2}$. Clouds have also an important impact on OLR, but this study focuses only on the clear-sky case.

Climate models contain fast approximate models for calculating OLR. However, to make sure that the fast models are correct one must make high resolution radiative transfer (RT) calculations with a precise line-byline RT model. This was first done by Shine and Sinha [4], using a model with $10 \mathrm{~cm}^{-1}$ frequency resolution, corresponding to 250 frequency grid points from 0 to $2500 \mathrm{~cm}^{-1}$. These calculations were considerably refined, firstly by Ridgway et al. [5], then by Clough et al. [6] and Clough and Iacomo [7], who used an adaptive frequency grid to achieve $0.2 \%$ computational accuracy. Such high resolution calculations can be used to study the sensitivity of the OLR in different frequency regions to perturbations in the humidity concentration at different altitudes. Fig. 2 shows the Jacobian of zenith monochromatic radiance with respect to humidity perturbations at different altitudes (see next section for exact definition). It shows a broad band of sensitivity to water vapor perturbations throughout the thermal infrared, interrupted only by the $\mathrm{CO}_{2}$ feature near $650 \mathrm{~cm}^{-1}$.

The calculations by Clough and coworkers included a better model of the water vapor continuum than earlier calculations. Due to the continuum, water vapor has a significant effect on OLR not only in the pure rotational band from approximately 0 to $600 \mathrm{~cm}^{-1}$ and the vibrational-rotational band from approximately 1400 to $2100 \mathrm{~cm}^{-1}$, but also in the continuum region between the bands, as evident from Fig. 2 . The figure also clearly shows that these different frequency regions of water vapor absorption are responsible for OLR sensitivity to water vapor perturbations at different altitudes, a fact first pointed out by Sinha and Harries [8], who particularly stressed the importance of the $0-500 \mathrm{~cm}^{-1}$ frequency region, where OLR is sensitive to perturbations in the mid- and upper troposphere.

This paper describes the implementation and validation of state-of-the-art continuum models for the public domain RT model ARTS [9], so that it can be used for OLR calculations. The continua implemented are 


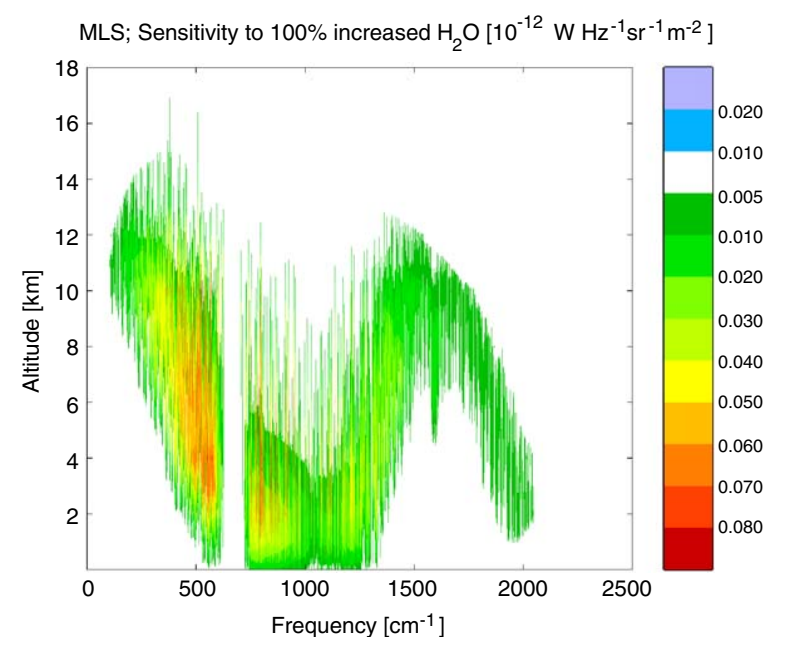

Fig. 2. The Jacobian of TOA zenith monochromatic radiance with respect to humidity in $\mathrm{W} \mathrm{Hz}^{-1} \mathrm{sr}^{-1} \mathrm{~m}^{-2}$ for a midlatitude summer (MLS) atmosphere. The units correspond to the OLR change for a doubling of the humidity concentration at one altitude, decreasing linearly to zero at the adjacent altitudes above and below (triangular perturbations). The grid spacing is $1 \mathrm{~km}$. The calculation was performed with the model and setup described in Section 2. The spectrum corresponding to this Jacobian is the one displayed in Fig. 1.

updated versions of the ones described by Clough and Iacomo [7], provided by Mlawer et al. [10]. Differences between the new ARTS OLR calculations and those of Clough and coworkers are also discussed. The paper is organized as follows: Section 2 presents the theoretical background and the model setup, including the atmospheric scenarios investigated, Section 3 results and discussion, and Section 4 summary and conclusions.

\section{Theoretical background and model setup}

\subsection{Basic assumptions and atmospheric scenarios}

Detailed line-by-line radiative transfer calculations were performed with the Atmospheric Radiative Transfer Simulator (ARTS), described in [9]. The model assumes a realistic spherical geometry for the atmosphere, which is an important difference to older models that assume a plane parallel atmosphere. Atmospheric scenarios were taken from the FASCOD scenarios [11]. They contain all trace gases required for the calculation. There are five different scenarios: tropical, midlatitude summer, midlatitude winter, subarctic summer, and subarctic winter.

The considered spectral range is from 0 to $2500 \mathrm{~cm}^{-1}$, similar to [7]. The most important radiatively active species in this spectral region are water vapor, carbon dioxide, methane, nitrous oxide, and ozone, with water vapor being by far the most important one. In addition to the line spectra, various continua have to be taken into account. Only the clear-sky case was considered. Clouds are known to have a very important impact on both the SW and the LW radiation, but, as stated above, are not the subject of this study. The surface emissivity was set to unity, following [6]. This should be a good approximation at infrared frequencies. The top of the atmosphere was assumed to be at $95 \mathrm{~km}$ where nothing else is stated.

As described in [9] the radiative transfer model ARTS can use different spectroscopic databases, among them HITRAN [12] and JPL [13]. For this study HITRAN was used. It lists about 1 million lines for 38 species between 0 and $2500 \mathrm{~cm}^{-1}$. Not all of these species are relevant for the calculated OLR, thus a species reduction was performed first. The reduced species list resulting from this procedure was: $\mathrm{H}_{2} \mathrm{O}$ (15190 lines), $\mathrm{CO}_{2}(38326$ lines), $\mathrm{O}_{3}$ (246426 lines), $\mathrm{N}_{2} \mathrm{O}$ (18343 lines), $\mathrm{CO}$ (1913 lines), $\mathrm{CH}_{4}$ (32071 lines), $\mathrm{O}_{2}$ (5114 lines), $\mathrm{NO}$ (12835 lines), $\mathrm{NO}_{2}$ (95005 lines), $\mathrm{HNO}_{3}$ (171504 lines), and $\mathrm{N}_{2}$ (106 lines). A comparison to OLR calculations covering all HITRAN species showed differences below $0.01 \%$ in OLR, while the number of lines was reduced by about 30\%. This line list was used for the calculations presented in Section 3. 


\subsection{OLR, Jacobian, and cooling rate calculation}

Following the notation of [6], the upwelling monochromatic radiative flux $F_{v}^{+}$and downwelling monochromatic radiative flux $F_{v}^{-}$at a given atmospheric level can be calculated from the monochromatic radiance $I_{v}$ integrated over all relevant propagation angles at that level as

$$
\begin{aligned}
& F_{v}^{+}(z)=\int_{\phi=0}^{2 \pi} \int_{\mu=0}^{1} I_{v}(z, \mu) \mu \mathrm{d} \mu \mathrm{d} \phi, \\
& F_{v}^{-}(z)=\int_{\phi=0}^{2 \pi} \int_{\mu=-1}^{0} I_{v}(z, \mu) \mu \mathrm{d} \mu \mathrm{d} \phi,
\end{aligned}
$$

where $\mu$ is the zenith angle cosine, $\phi$ the azimuth angle, and $v$ the frequency. The units of the monochromatic radiative flux $F_{v}$ are $\mathrm{W} \mathrm{m}^{-2} \mathrm{~Hz}^{-1}$, those of the monochromatic radiance $I_{v}$ are $\mathrm{W} \mathrm{m}^{-2} \mathrm{~Hz}^{-1} \mathrm{sr}^{-1}$. The integration over $\mu \mathrm{d} \mu$ is equal to an integration over $\cos (\theta) \sin (\theta) \mathrm{d} \theta$, where $\theta$ is the zenith angle. The term $\cos (\theta)$ is a result of the projection onto the zenith direction, since only the radiance component perpendicular to the azimuthal plane contributes to the flux. The remaining parts result from the azimuthal integration. Since radiances are assumed to be azimuthally independent the azimuthal integration is trivial and can be carried out directly, leading to

$$
F_{v}^{+}(z)=2 \pi \int_{0}^{1} I_{v}(z, \mu) \mu \mathrm{d} \mu
$$

and a similar equation for the downwelling monochromatic radiative flux.

The total upwelling radiative flux $F^{+}$can be calculated easily by integrating (3) over frequency, leading to

$$
\begin{aligned}
F^{+}(z) & =\int_{0}^{\infty} F_{v}^{+}(z) \mathrm{d} v \\
& =2 \pi \int_{0}^{\infty} \int_{0}^{1} I_{v}(z, \mu) \mu \mathrm{d} \mu \mathrm{d} v \\
& =2 \pi \int_{0}^{1} I(z, \mu) \mu \mathrm{d} \mu,
\end{aligned}
$$

where $I$ in the last row is the (total) radiance in $\mathrm{W} \mathrm{m}^{-2} \mathrm{sr}^{-1}$. Finally, the net radiative flux is obtained by taking the difference between upwelling and downwelling contributions,

$$
F(z)=F_{v}^{+}(z)-F_{v}^{-}(z)
$$

Note that the direction of positive fluxes is upwards.

The upwelling radiative flux $F^{+}$at the top of the atmosphere represents the OLR. The upwelling and downwelling radiative fluxes at the tropopause level are also of interest, since the main impact of water vapor on the OLR comes from the troposphere (see [6] for a more detailed discussion).

The spectrum of monochromatic radiance $I_{v}$ is the quantity calculated internally by the RT model. If one wants to see how sensitive this is to variations in the atmospheric state, the suitable quantity to study is the derivative of $I_{v}$ with respect to changes in the concentration of gases such as water vapor. This quantity,

$$
\mathbf{K}=\frac{\partial \mathbf{y}}{\partial \mathbf{x}}
$$

is called the Jacobi matrix, or Jacobian, where $\mathbf{y}$ is a vector of $I_{v}$ for different frequencies and $\mathbf{x}$ is the vector describing the atmospheric state. ARTS can calculate Jacobians analytically for trace gas concentrations and semi-analytically for temperature [9]. Different options for the units of the Jacobian calculation are available in ARTS. For this study fractional VMR units were used, which means that the Jacobians give the sensitivity to relative changes in the trace gas VMR. Jacobians of monochromatic radiance can be integrated over frequency to obtain Jacobians of total radiance.

Another important quantity is the cooling rate, defined as the rate of temperature change of an atmospheric layer due to loss of energy by emission of radiation. Of course, this includes also the possibility of heating by 
absorption of radiation, which leads to a negative cooling rate. Heating occurs primarily by absorption of solar SW radiation, while cooling occurs primarily by emission of LW radiation. Only the latter part is considered here.

The cooling rate, $C$, can be calculated as

$$
C(z) \equiv-\frac{\mathrm{d} T(z)}{\mathrm{d} t}=\frac{1}{\rho(z) c_{p}} \frac{\mathrm{d} F(z)}{\mathrm{d} z},
$$

where $T$ is the temperature, $t$ the time, $\rho$ the air density, $c_{p}$ the heat capacity (for pressure work), $F$ the net radiative flux and $z$ the altitude. By inserting the definition of $F$ one can rewrite this to obtain the cooling rate directly from the monochromatic radiance $I_{v}$ :

$$
C(z)=\frac{2 \pi}{\rho(z) c_{p}} \int_{v=0}^{\infty} \int_{\theta=0}^{\pi}\left[B_{v}(T(z))-I_{v}(z, \theta)\right] \alpha(z) \sin (\theta) \mathrm{d} \theta \mathrm{d} v,
$$

where $B_{v}(T(z))$ is the Planck function and $\alpha(z)$ is the absorption coefficient.

One can see from this equation that the contribution to the cooling rate will be zero for frequencies where $\alpha=0$.

To obtain correct results it is crucial that $I$ converges to $B_{v}$ when it is expected that $I=B_{v}$, which is the case when the absorption is very high. The used forward model, ARTS, uses a straightforward scheme for performing the radiative transfer. This scheme uses the mean value of the Planck function, at the end points of the radiative transfer integration step, as the effective source function. This averaging is theoretically only correct for cases where the optical thickness is zero. A more elaborate scheme would put more emphasis on the Planck function value for the integration step end-point close to the sensor and less emphasis on the Planck function value for the integration step end-point far from the sensor. In cases of extremely high absorption, the effective source function would be equal to the Planck function at the end-point close to the sensor. Such more advanced schemes have been tested for ARTS but were found to give no practical improvements for simulation of remote sensing measurements, the main objective of ARTS. The calculation accuracy in ARTS is to a large extent controlled by varying the length of the radiative transfer step length and it was found that it was more effective, considering calculation time, to decrease the step length than to use a more advanced expression to solve the radiative transfer.

Considering that radiative transfer applies a mean value of the Planck function at the end points of the integration step, it is not a good idea to compare $I$ with the $B_{v}$ for the position of interest. This would require an extremely short radiative transfer step length, a statement verified by practical calculations. A better solution is to replace $\left(B_{v}(T(z))-I(z, \theta)\right)$ in $(8)$ by $(\Psi-I(z, \theta))$, where $\Psi$ is the effective source function for the radiative transfer step closest to the point of interest. This modification balances the radiation budget perfectly for high values of $\alpha$ and has a small impact on the accuracy.

\subsection{Absorption calculation}

Absorption coefficients were calculated line-by-line with the $\mathrm{C}++$ code ARTS, assuming a Voigt [14] lineshape with a cutoff at a distance of $25 \mathrm{~cm}^{-1}$ from the line center. The cutoff is performed as described in [15]. For species that show continuum absorption, i.e., $\mathrm{H}_{2} \mathrm{O}, \mathrm{CO}_{2}, \mathrm{O}_{2}$, and $\mathrm{N}_{2}$, semi-empirical continua from the MT_CKD_1.00 FORTRAN code by Mlawer, Tobin, Clough, Kneizys, and Davies [10] were added, which were translated to $\mathrm{C}++$.

For $\mathrm{H}_{2} \mathrm{O}$ the calculation includes the self- and foreign far-wing continua throughout the considered measurement range. For $\mathrm{N}_{2}$ the calculation includes the roto-translational collision-induced absorption band according to Borysow and Frommhold [16] in the $0-350 \mathrm{~cm}^{-1}$ wavenumber range, and the fundamental collision induced absorption band according to Lafferty et al. [17] in the $2085-2670 \mathrm{~cm}^{-1}$ wavenumber range. For $\mathrm{O}_{2}$ the calculation includes the fundamental collision induced absorption band according to Thibault et al. [18] in the $1340-1850 \mathrm{~cm}^{-1}$ wavenumber range. Finally, for $\mathrm{CO}_{2}$ the calculation includes the far wing continuum according to Kneizys et al. [19] throughout the considered wavenumber range. There was no attempt to account for $\mathrm{CO}_{2}$ line mixing effects. 
To validate the ARTS absorption model we participated in the AIRS RT model intercomparison organized by the International TOVS Study Group (ITWG), a follow-up activity of the TOVS RT model intercomparison described by Garand et al. [20]. Compared were simulated Atmospheric Infrared Sounder (AIRS) radiances in the $650-2700 \mathrm{~cm}^{-1}$ wavenumber range. Averaged over the 52 different intercomparison scenarios, ARTS has a mean bias of only $-0.06 \mathrm{~K}$ and mean standard deviation of $0.27 \mathrm{~K}$ against the so-called Reference Forward Model (RFM) which is based on the GENLN2 model [21]. The agreement is significantly better in the spectral regions dominated by water vapor, differences there are caused primarily by different continuum implementations. In the spectral regions dominated by $\mathrm{O}_{3}$ and $\mathrm{CO}_{2}$ ARTS has a slight cold bias of about $-0.2 \mathrm{~K}$.

\subsection{Frequency and zenith angle grids}

The numerical evaluation of (4) requires the calculation of monochromatic radiances $I_{v}(\mu)$ at a discrete set of frequencies $v$ and zenith angle cosines $\mu$.

Different frequency grid options were investigated, on one hand equidistant grids (EG) with varying density, on the other hand non-equidistant grids chosen by different strategies. The two strategies tried were minimization of absolute deviation ('optimized grid deviation', OGD) and minimization of absolute area deviation ('optimized grid area deviation', OGAD). The OGD strategy starts out from a very coarse grid. The frequency point where the linearly interpolated monochromatic radiance has the largest absolute deviation from a high resolution reference calculation is determined and added to the frequency grid vector. This procedure is repeated until the maximum deviation is below the desired threshold. The OGAD strategy works in the same way, with the difference that the new frequency grid points are added where the absolute area deviation between interpolated and reference monochromatic radiance is largest.

The results of the frequency grid investigation are summarized in Fig. 3, which shows simulated total zenith radiance, as defined in the last row of (4), at the top of the atmosphere as a function of the number of frequency grid points. For the full investigated frequency range $\left(0-2500 \mathrm{~cm}^{-} 1\right)$, EG calculations were made for grids with 1000, 10,000, 20,000 and 40,000 points. This represents grid spacings of 2.5, 0.25, 0.125, and $0.063 \mathrm{~cm}^{-1}$, respectively. Although these grids are rather coarse, convergence is already achieved with these resolutions. Convergence was tested by comparing the simulated radiance to the reference case, which was calculated with a grid of 500,000 points, corresponding to a resolution of $0.005 \mathrm{~cm}^{-1}$.

The OGD method was used to produce three different grids with maximum deviations set to $5.0 \mathrm{e}-14$, $2.5 \mathrm{e}-14$, and $1.0 \mathrm{e}-14 \mathrm{~W} \mathrm{~m}^{-2} \mathrm{sr}^{-1} \mathrm{~Hz}^{-1}$, resulting in grid sizes of $19,760,34,184$, and 57,522 points. The OGAD

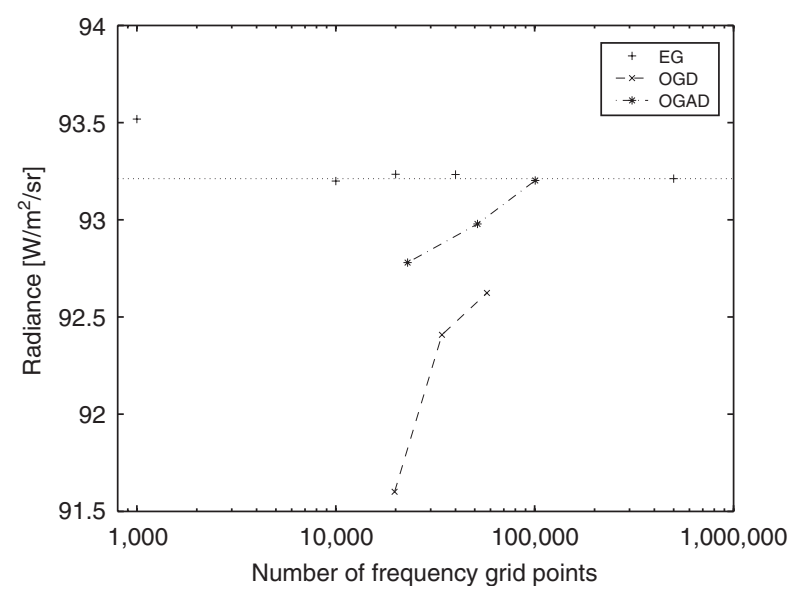

Fig. 3. Results of the grid investigation. The horizontal axis shows the total number of frequency grid points in logarithmic scale, the vertical axis the associated simulated total radiance. The calculations were carried out for a mid-latitude summer scenario. The ' + ' symbols mark the equidistant grid (EG) cases, the EG case with 500,000 grid points was taken as the reference to judge the other cases. The ' $X$ ' and ' $*$ ' symbols mark the OGD and OGAD cases, respectively. 


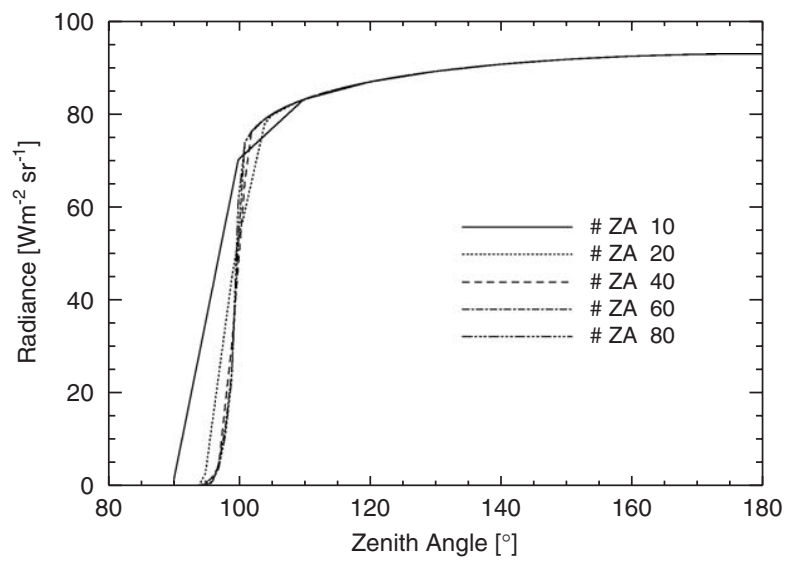

Fig. 4. Radiance at the top of the atmosphere as a function of zenith angle for different zenith angle grids. The number of grid points for each case is given in the legend. The atmospheric scenario is mid-latitude summer, the same as in Fig. 3.

Table 1

Mid-latitude summer OLR (upwelling radiative flux $F^{+}$at the top of the atmosphere) as a function of the number of points in the zenith angle (ZA) grid

\begin{tabular}{llr}
\hline ZA points & OLR $\left(\mathrm{W} \mathrm{m}^{-2}\right)$ & Diff. to max. ZA $(\%)$ \\
\hline 10 & 274.37 & 0.93 \\
20 & 272.03 & 0.07 \\
40 & 271.61 & -0.08 \\
60 & 271.72 & -0.04 \\
80 & 271.83 & 0.0 \\
\hline
\end{tabular}

The last column gives the relative difference with respect to the case with $80 \mathrm{ZA}$ grid points.

method was used to produce three grids with grid sizes of 22,950, 51,612, and 100,674 points. For all investigated methods the simulated radiances converge to the reference case with increasing number of frequency grid points. However, interestingly, both the OGD and OGAD method perform poorly compared to the simple EG method. The explanation for this unexpected result is that the equidistant grids lead to a quasi-random sampling of spectral line features, due to the large density of spectral lines in the thermal infrared. Thus, positive and negative errors for the equidistant grids cancel to a large extent.

It should be noted that the performance of the OGD and OGAD method could most likely be improved considerably by using higher order interpolation methods. However, the performance of the much simpler EG method was so good that it was decided not to pursue this question further. The equidistant grid with 10,000 frequency grid points was adopted for the calculations discussed in Section 3.

The other grid that has to be addressed here is the zenith angle grid. Fig. 4 shows the simulated radiance at the top of the atmosphere as a function of zenith angle for equidistant zenith angle grids of different resolution. The major variations in radiance are happening near the limb at angles below $110^{\circ}$. Calculated OLR values ( $F^{+}$at $95 \mathrm{~km}$ altitude) for a mid-latitude summer atmospheric scenario for different zenith angle grid densities are shown in Table 1. As one can see from Fig. 4, the radiance variation over zenith angles is very smooth, thus even a small number as 10 angles already yields fairly accurate results. A total number of 40 zenith angles was judged to be adequate for the present study, leading to an accuracy below $0.1 \%$. Further optimization could probably be achieved by using a non-equidistant zenith angle grid, if necessary. 


\section{Results and discussion}

\subsection{Fluxes and cooling rates}

Table 2 shows calculated fluxes $F^{+}$at the tropopause (TP) and at the TOA for the five FASCOD scenarios: tropical (TRO), midlatitude summer (MLS), midlatitude winter (MLW), subarctic summer (SAS), and subarctic winter (SAW). Results of Clough and Iacomo [7], hereafter referred to as CLI, for the TRO, MLS, and MLW scenario are also shown. The tropopause altitudes of our calculations were the same as those of CLI for these scenarios.

Interestingly, our results at the TOA consistently show a low bias of approximately $4.1 \%$ against the CLI results. Different possible reasons for this discrepancy were checked by control calculations for the midlatitude summer scenario. Removing the water vapor continuum completely increases the OLR by only $2.8 \%$, so differences in the water vapor continuum alone cannot explain the bias. The small difference in the assumed atmospheric state leads to an OLR difference of approximately $0.5 \%$, but in the wrong direction. Restricting the species selection to only those used by CLI increases the OLR only by $0.1 \%$. Changing the calculation frequency range from $0-2500$ to $10-3000 \mathrm{~cm}^{-1}$, as in the CLI calculation, increases the OLR by $0.2 \%$. Decreasing the sensor altitude from 95 to $60 \mathrm{~km}$ increases the OLR by $1.1 \%$ (CLI used $65 \mathrm{~km}$ ). However, the biggest difference is caused by the plane parallel approximation, which was made in the CLI calculation but not in our calculation. A control run with plane parallel approximation increased $F^{+}$at $95 \mathrm{~km}$ by $2.5 \%$ to $278.6 \mathrm{~W} \mathrm{~m}^{-2}$. The remaining difference is likely to be caused by differences in the water vapor and dry air continua, as well as the spectroscopic database. As shown elsewhere [22,27], both the CLI calculations and our calculations are in reasonable agreement with satellite based clear-sky OLR measurements by the CERES/ TRMM instrument: the CLI results lie on the upper end of the CERES clear-sky OLR variability range, whereas our results lie on the lower end.

Fig. 5 shows the calculated spectral cooling rates for the MLS scenario. The figure can be directly compared to Plate 1 of CLI. The overall agreement is excellent, with the exception of a negative cooling rate feature near $1300 \mathrm{~cm}^{-1}$ in frequency and near $200 \mathrm{hPa}$ in pressure. Important trace gases in this frequency region are $\mathrm{N}_{2} \mathrm{O}$ and $\mathrm{CH}_{4}$, judged from the Jacobians of these species (not shown). It seems that this feature is missing in the CLI calculations which may explain part of the bias in OLR. Unfortunately, an explicit check of this hypothesis is not possible, since we do not have the exact line list that was used in the CLI calculation.

\subsection{Sensitivity to humidity, carbon dioxide, and temperature changes}

The FASCOD scenarios can be used to visualize the impact of large scale changes of humidity, carbon dioxide, and temperature. For this purpose modified profiles were generated. The modifications were:

Table 2

Calculated radiative fluxes for the FASCOD scenarios

\begin{tabular}{|c|c|c|c|c|c|}
\hline Scenario & TP Alt (km) & $F^{+}(\mathrm{TP})$ & $F^{-}(\mathrm{TP})$ & $F(\mathrm{TP})$ & $F^{+}(\mathrm{TOA})$ \\
\hline TRO & 17.0 & 285.4 & -11.0 & 274.4 & 278.0 \\
\hline TRO CLI & & 289.3 & -11.7 & 277.6 & 290.3 \\
\hline MLS & 13.0 & 283.2 & -21.6 & 261.6 & 271.6 \\
\hline MLS PP & & 283.4 & -21.7 & 261.7 & 278.6 \\
\hline MLS CLI & & 287.6 & -22.3 & 265.3 & 283.3 \\
\hline MLW & 10.0 & 237.4 & -30.4 & 207.0 & 222.9 \\
\hline MLW CLI & & 238.3 & -25.1 & 213.2 & 232.6 \\
\hline SAS & 10.0 & 268.3 & -37.0 & 231.3 & 254.6 \\
\hline SAW & 09.0 & 205.2 & -32.4 & 172.8 & 193.4 \\
\hline
\end{tabular}

See text for definition of scenario acronyms. Shown are the tropopause altitude, the upwelling-, downwelling-, and net flux at the tropopause, and the upwelling flux at the TOA. All fluxes are in $\mathrm{W} \mathrm{m}^{-2}$. For each class there is one row for our calculation and a second row for the CLI calculation. For the MLS class there is also a row 'MLS PP' which shows our calculation for a plane parallel atmosphere. Note that CLI assume the TOA to be at $65 \mathrm{~km}$, whereas we assume it to be $95 \mathrm{~km}$. 


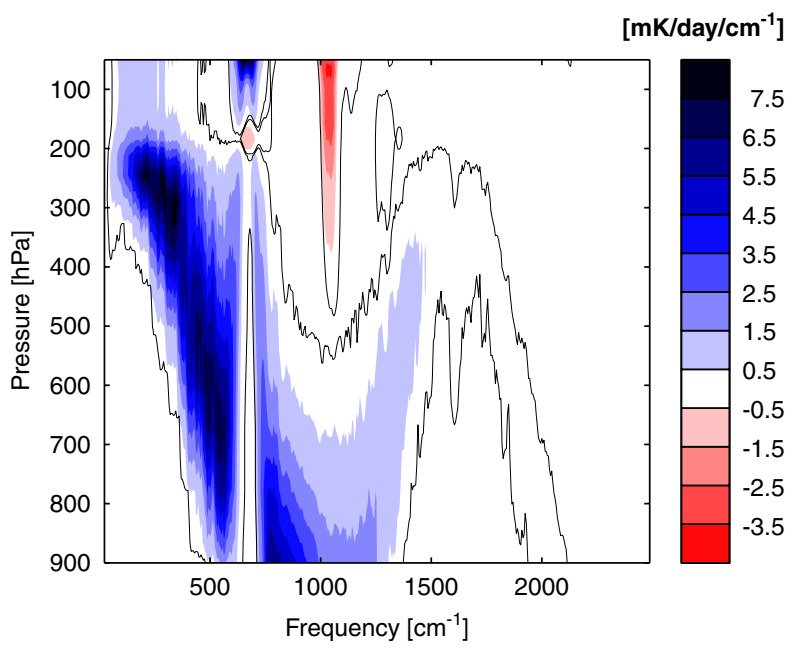

Fig. 5. Spectral cooling rates for the FASCOD MLS scenario. The results are averaged in frequency with a boxcar filter of width $25 \mathrm{~cm}^{-1}$. Black contour levels indicate $\pm 0.1 \mathrm{mK} \mathrm{day}^{-1} \mathrm{~cm}$.

(a) water vapor mixing ratio increased by $20 \%$ throughout the column, (b) water vapor mixing ratio decreased by $20 \%$ throughout the column, (c) carbon dioxide mixing ratio increased by $100 \%$ throughout the column (double- $\mathrm{CO}_{2}$ ), (d) temperature increased by $1 \mathrm{~K}$ throughout the column keeping absolute humidity fixed, and (e) temperature increased by $1 \mathrm{~K}$ throughout the column keeping relative humidity fixed. The rationale for this set of modifications was to compare the sensitivity of OLR to humidity changes to the sensitivity to carbon dioxide and temperature changes.

The results are shown in Fig. 6 for the TRO case (top) and the SAW case (bottom). In the TRO case the $20 \%$ humidity increase has a larger impact on OLR than the $\mathrm{CO}_{2}$ doubling, in the SAW case the $\mathrm{CO}_{2}$ doubling has the slightly larger impact. This is consistent with the findings of Brindley and Harries [23], who stated that humidity increases of $12 \%$ and $25 \%$, for the TRO and SAW cases, respectively, have an OLR impact equivalent to doubling $\mathrm{CO}_{2}$. However, OLR is also strongly sensitive to changes in temperature because of the positive temperature dependence of the Planck function. The ' $T+1 \mathrm{~K}$ ' bar in Fig. 6 shows that for the TRO case a $1 \mathrm{~K}$ temperature increment throughout the atmosphere produces roughly the same effect on OLR as a $20 \%$ humidity decrease. For the SAW case the $+1 \mathrm{~K}$ temperature effect is even roughly twice the $-20 \%$ humidity effect. Because of the partial cancellation of OLR changes due to a simultaneous increase of temperature and absolute humidity, the OLR sensitivity to a $1 \mathrm{~K}$ temperature increase under fixed relative humidity is much smaller than to a pure temperature increase. This is the so-called water vapor feedback. Luckily, the net OLR impact of a temperature change with fixed relative humidity is still positive, otherwise we would be in a situation of runaway greenhouse effect (see [24] for a more detailed discussion).

The altitude region where OLR is sensitive to humidity changes is shown in Fig. 7. Here Jacobians for monochromatic radiance (see Fig. 2) were spectrally integrated. This was done for all five FASCOD scenarios. The figure shows that humidity changes throughout the free troposphere are important. Particularly, the region of sensitivity reaches up to pressures below $400 \mathrm{hPa}$ (approximately $7 \mathrm{~km}$ ), where the available humidity information on a global scale is rather poor. In the case of the TRO scenario, the Jacobian shows a characteristic two-peaked structure, which was already noticed by Sinha and Allen [25]. Note that the presence of clouds will further emphasize the upper troposphere compared to this clear-sky calculation, as shown by Colman [26]. The figure shows also that temperature changes are important throughout the free troposphere.

\section{Summary and conclusions}

The Atmospheric Radiative Transfer Simulator (ARTS), a high frequency resolution radiative transfer model, was used to simulate the clear-sky outgoing longwave radiation (OLR) flux, Jacobians for the clear-sky monochromatic zenith radiance, and monochromatic clear-sky cooling rates. Compared to the simulations by 

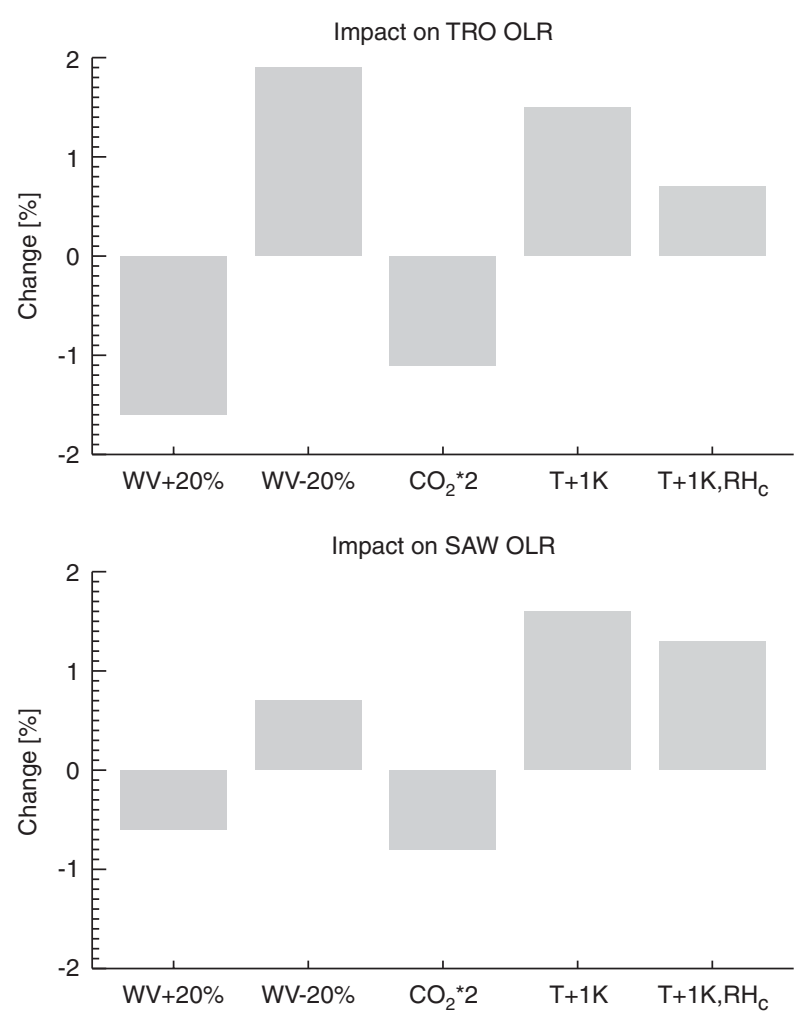

Fig. 6. OLR impact of a $20 \%$ water vapor increase or decrease, $\mathrm{CO}_{2}$ doubling, a $1 \mathrm{~K}$ increase in temperature with fixed absolute humidity, and a $1 \mathrm{~K}$ increase in temperature with fixed relative humidity. Results are shown for the TRO scenario (top) and the SAW scenario (bottom). Shown is the relative deviation in $\operatorname{OLR}\left(F^{+}\right.$at $95 \mathrm{~km}$ altitude) from the reference case.
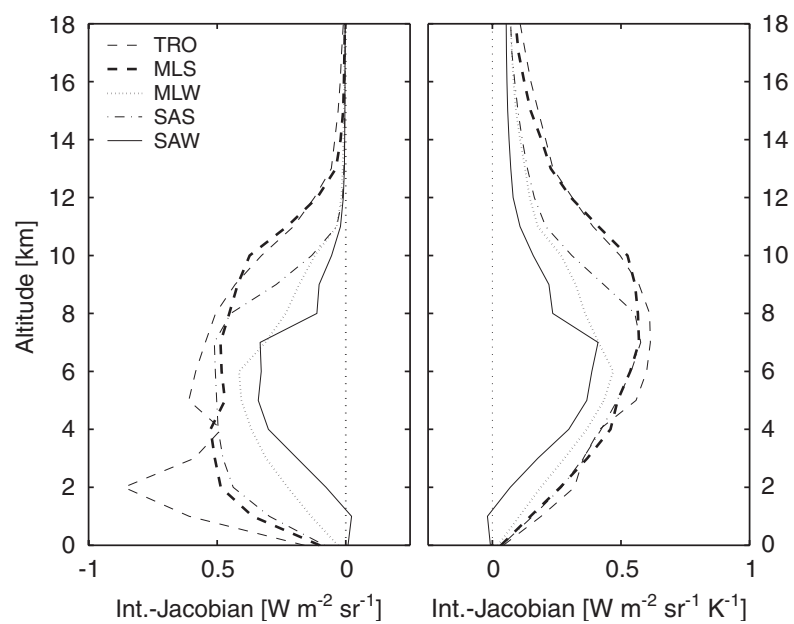

Fig. 7. Jacobians with respect to humidity (left) and temperature (right) for total zenith radiance at $95 \mathrm{~km}$ altitude for the five different FASCOD scenarios. The humidity Jacobian is for a fractional change in humidity relative to the reference case at one grid point, decreasing linearly to zero at the adjacent grid points. The unit corresponds to the change in radiance for a doubling of the water vapor concentration. For temperature, the unit corresponds to the change in radiance for a $1 \mathrm{~K}$ temperature change. The grid spacing is $1 \mathrm{~km}$.

Clough and Iacomo [7] (CLI) the model used updated spectroscopic data from the current version of HITRAN [12] and updated continuum parameterizations provided by Mlawer et al. [10]. With these settings ARTS was shown to be a valuable tool for the calculation of precise OLR values. 
It was shown that ultra-high frequency resolution is not needed for equidistant frequency grids, due to the quasi-random sampling of spectral line features leading to cancellation of positive and negative errors. The deviation of a calculation with only 1000 frequency grid points from the reference calculation with 500,000 frequency grid points is less than $2.0 \mathrm{~W} \mathrm{~m}^{-2}$. For 'optimized' non-equidistant frequency grids more grid points are needed to achieve a similar accuracy.

Compared to the calculations by CLI, our OLR at the TOA is approximately $4.1 \%$ smaller for all investigated scenarios. This is partly due to the fact that CLI assumed the TOA to be at $65 \mathrm{~km}$ altitude and made a plane parallel approximation, whereas we assume the TOA to be at $95 \mathrm{~km}$ altitude and do not make a plane parallel approximation. The combined effect of these two differences in setup explains a difference of approximately $2.6 \%$. The remaining $1.7 \%$ difference is probably due to the differences in spectroscopy and continuum models, since all other likely explanations were excluded. As shown elsewhere [22,27], both the CLI calculations and our calculations are in reasonable agreement with satellite based clear-sky OLR measurements by the CERES/TRMM instrument: the CLI results lie on the upper end of the CERES clear-sky OLR variability range, whereas our results lie on the lower end.

The mean value of clear-sky OLR most strongly depends on the mean temperature and humidity profile. A $20 \%$ change in humidity has a larger impact on the OLR than a doubling of the $\mathrm{CO}_{2}$ concentration for the TRO case, and an almost equal impact as double $\mathrm{CO}_{2}$ for the SAW case. The sensitive altitude region for temperature and humidity changes is the entire free troposphere, including the upper troposphere where humidity data quality is poor.

\section{Acknowledgements}

Thanks to Atmospheric and Environmental Research, Inc., in particular T. Clough and E. Mlawer, for making the latest continuum models publicly available at http://www.rtweb.aer.com/continuum_frame.html. Furthermore, thanks to the ARTS radiative transfer community, many of whom have indirectly contributed by implementing features to the ARTS model.

This study was funded by the German Federal Ministry of Education and Research (BMBF), within the AFO2000 project UTH-MOS, Grant 07ATC04, and by the European Space Agency (ESA) within project ACECLIM, ESTEC Contract No. $17479 / 03 / \mathrm{NL} / \mathrm{FF}$. It is a contribution to COST Action 723 'Data Exploitation and Modeling for the Upper Troposphere and Lower Stratosphere'.

\section{References}

[1] Salby ML. Fundamentals of atmospheric physics, International geophysics series, vol. 61. New York: Academic Press; 1996.

[2] Harries JE. The greenhouse earth: a view from space. Q J R Meteorol Soc 1996;122:799-818.

[3] Harries JE. Atmospheric radiation and atmospheric humidity. Q J R Meteorol Soc 1997;123:2173-86.

[4] Shine KP, Sinha A. Sensitivity of the earth's climate to height-dependent changes in the water vapour mixing ratio. Nature 1991;354:382-4.

[5] Ridgway WL, Harshvardhan, Arking A. Computation of atmospheric cooling rates by exact and approximate methods. J Geophys Res 1991;96(D5):8969-84.

[6] Clough SA, Iacono MJ, Moncet J-L. Line-by-line calculations of atmospheric fluxes and cooling rates: application to water vapor. J Geophys Res 1992;97(D14):15761-85.

[7] Clough SA, Iacomo MJ. Line-by-line calculation of atmospheric fluxes and cooling rates. 2. Application to carbon dioxide, ozone, methane, nitrous oxide and the halocarbons. J Geophys Res 1995;100(D8):16519-35.

[8] Sinha A, Harries JE. Water vapor and greenhouse trapping - the role of far-infrared absorption. J Geophys Res 1995;22:2147-50.

[9] Buehler SA, Eriksson P, Kuhn T, von Engeln A, Verdes C. ARTS, the atmospheric radiative transfer simulator. JQSRT 2005;91(1):65-93.

[10] Mlawer MJ, Tobin DC, Clough SA. A revised perspective on the water vapor continuum: the MT_CKD model. JQSRT 2004, in preparation [URL: http: //www.rtweb.aer.com/continuum_frame.html].

[11] Anderson GP, Clough SA, Kneizys FX, Chetwynd JH, Shettle EP. AFGL atmospheric constituent profiles (0-120 km). Technical Report TR-86-0110, AFGL; 1986.

[12] Rothman LS, Barbe A, Benner DC, Brown LR, Camy-Peyret C, Carleer MR, Chance K, Clerbaux C, Dana V, Devi VM, Fayt A, Flaud J-M, Gamache RR, Goldman A, Jacquemart D, Jucks KW, Lafferty WJ, Mandin J-Y, Massie ST, Nemtchinov V, Newnham DA, Perrin A, Rinsland CP, Schroeder J, Smith KM, Smith MAH, Tang K, Toth RA, Auwera JV, Varanasi P, Yoshino K. The HITRAN molecular spectroscopic database: edition of 2000 including updates of 2001. JQSRT 2003;82 [HITRAN special issue]. 
[13] Pickett HM, Poynter RL, Cohen EA. Submillimeter, millimeter, and microwave spectral line catalogue. Technical Report JPL Publication 80-23, Rev. 3, Jet Propulsion Laboratory; 1992.

[14] Kuntz M. A new implementation of the humlicek algorithm for the calculation of the voigt profile function. JQSRT 1997;57(6):819-24.

[15] Clough SA, Kneizys FX, Davies RW. Line shape and the water vapor continuum. Atmos Res 1989;23:229-41.

[16] Borysow A, Frommhold L. Collision induced rototranslational absorption spectra of $\mathrm{N}_{2}-\mathrm{N}_{2}$-pairs for temperatures from 50 to $300 \mathrm{~K}$. Astrophys J 1986;311:1043-57 erratum: 320, 437, 1987. Electronic reprint provided by the NASA Astrophysical Data System.

[17] Lafferty WJ, Solodov AM, Weber A, Olsen WB, Hartmann JM. Infrared collision-induced absorption by $\mathrm{N}_{2}$ near $4.3 \mu \mathrm{m}$ for atmospheric applications: measurements and empirical modeling. Appl Opt 1996;35:5911-7.

[18] Thibault F, Moreau G, Doucen RL, Rosenmann L, Hartmann J-M, Boulet C. Infrared collision-induced absorption by $\mathrm{O}_{2}$ near $6.4 \mu \mathrm{m}$ for atmospheric applications: measurements and empirical modeling. Appl Opt 1997;36:563-7.

[19] Kneizys FX, Abreu LW, Anderson GP, Chetwynd JH, Shettle EP, Berk A, Bernstein LS, Robertson DC, Acharya P, Rothman LS, Selby JEA, Gallery WO, Clough SA. The MODTRAN2/3 report and LOWTRAN7 model laboratory studies and propagation modeling. Technical Report 1/11/96, Phillips Laboratory, Geophysical Directorate, PL/GPOS; 1996 [editors: Abreu LW, Anderson GP].

[20] Garand L, et al. Radiance and jacobian intercomparison of radiative transfer models applied to HIRS and AMSU channels. J Geophys Res 2001;106(D20):24017-31.

[21] Edwards DP. GENLN2: a general line-by-line atmospheric transmittance and radiance model. Technical Report NCAR/ TN367+STR, National Center for Atmospheric Research, Boulder, Colorado; 1992.

[22] Buehler SA, John VO, von Engeln A, Brocard E, Kuhn T, Eriksson P. Understanding the global variability of clear-sky outgoing longwave radiation. Q J R Meteorol Soc 2005, submitted for publication [preprint available at www. sat. uni-bremen. de].

[23] Brindley HE, Harries JE. The impact of far I.R. absorption on clear sky greenhouse forcing: sensitivity study at high spectral resolution. JQSRT 1998;60(2):151-80.

[24] Held IM, Soden BJ. Water vapor feedback and global warming. Annu Rev Energy Environ 2000;25:441-75.

[25] Sinha A, Allen MR. Climate sensitivity and tropical moisture distribution. J Geophys Res 1994;99(D2):3707-16.

[26] Colman RA. On the vertical extent of atmospheric feedbacks. Climate Dyn 2001;17:391-405.

[27] Buehler SA. Remote sensing of atmospheric composition for climate applications. Habilitationschrift, University of Bremen, 2004 (Weblink: http://www.sat.unibremen.de/members/sab/publications/habil/). 